\title{
Identification of the anti-tumor activity and mechanisms of nuciferine through a network pharmacology approach
}

\author{
Quan $\mathrm{QI}^{1,2, \#}$, Rui $\mathrm{LI}^{1,2, \#}$, Hui-ying $\mathrm{LI}^{2}$, Yu-bing $\mathrm{CAO}^{1}$, Ming BAl${ }^{2}$, Xiao-jing $\mathrm{FAN}^{3}$, Shu-yan WANG${ }^{4}$, Bo ZHANG ${ }^{2,4, *}$, Shao $\mathrm{LI}^{2, *}$ \\ ${ }^{1}$ Tianjin State Key Laboratory of Modern Chinese Medicine, Tianjin University of Traditional Chinese Medicine, Tianjin 300193, \\ China; ${ }^{2}$ MOE Key Lab of Bioinformatics, Bioinformatics Division, TNLIST and Department of Automation, Tsinghua University, Beijing \\ 100084, China; ${ }^{3}$ The Second Hospital Affiliated to Tianjin University of Traditional Chinese Medicine, Tianjin 300150, China; ${ }^{4}$ Tianjin \\ International Joint Academy of Biotechnology \& Medicine, Tianjin 300457, China
}

Aim: Nuciferine is an aporphine alkaloid extracted from lotus leaves, which is a raw material in Chinese medicinal herb for weight loss. In this study we used a network pharmacology approach to identify the anti-tumor activity of nuciferine and the underlying mechanisms.

Methods: The pharmacological activities and mechanisms of nuciferine were identified through target profile prediction, clustering analysis and functional enrichment analysis using our traditional Chinese medicine (TCM) network pharmacology platform. The antitumor activity of nuciferine was validated by in vitro and in vivo experiments. The anti-tumor mechanisms of nuciferine were predicted through network target analysis and verified by in vitro experiments.

Results: The nuciferine target profile was enriched with signaling pathways and biological functions, including "regulation of lipase activity", "response to nicotine" and "regulation of cell proliferation". Target profile clustering results suggested that nuciferine to exert anti-tumor effect. In experimental validation, nuciferine $(0.8 \mathrm{mg} / \mathrm{mL})$ markedly inhibited the viability of human neuroblastoma SY5Y cells and mouse colorectal cancer CT26 cells in vitro, and nuciferine $(0.05 \mathrm{mg} / \mathrm{mL})$ significantly suppressed the invasion of 6 cancer cell lines in vitro. Intraperitoneal injection of nuciferine $(9.5 \mathrm{mg} / \mathrm{mL}$, ip, 3 times a week for 3 weeks) significantly decreased the weight of SY5Y and CT26 tumor xenografts in nude mice. Network target analysis and experimental validation in SY5Y and CT26 cells showed that the anti-tumor effect of nuciferine was mediated through inhibiting the PI3K-AKT signaling pathway and IL-1 levels in SY5Y and CT26 cells.

Conclusion: By using a TCM network pharmacology method, nuciferine is identified as an anti-tumor agent against human neuroblastoma and mouse colorectal cancer in vitro and in vivo, through inhibiting the PI3K-AKT signaling pathways and IL-1 levels.

Keywords: nuciferine; TCM network pharmacology; anti-tumor agent; neuroblastoma; colorectal cancer; PI3K-AKT; IL-1; drugCIPHER

Acta Pharmacologica Sinica (2016) 37: 963-972; doi: 10.1038/aps.2016.53; published online 16 May 2016

\section{Introduction}

Natural small-molecule compounds in Traditional Chinese Medicine (TCM) can serve as a continuous source for novel anti-tumor drug discovery. Currently, some of the natural compounds have been reported to exert anti-tumor activities, and they either have potential for development or have already been developed as antineoplastic drugs (eg, paclitaxel and arsenic trioxide, as well as artemisinin and its deriva-

\footnotetext{
\# These two authors contributed equally to this work.

* To whom correspondence should be addressed.

E-mail shaoli@mail.tsinghua.edu.cn (Shao LI); z-b07@mails.tsinghua.edu.cn (Bo ZHANG)

Received 2015-11-25 Accepted 2016-01-19
}

tives $)^{[1-3]}$. However, the traditional trial-and-error methods used to identify active compounds from numerous herbal compounds in TCM are time-consuming and labor intensive. Additionally, modern high-throughput screening assays have limitations in identifying the active compounds from TCM herbs because many herbal compounds feature weak or moderate binding to multiple targets ${ }^{[4,5]}$, even though TCM formulas have been shown to inhibit tumors in clinical settings ${ }^{[6]}$. Therefore, research regarding the identification of potentially active compounds from TCMs might require novel approaches, such as emerging TCM network pharma$\operatorname{colog} y^{[7,8]}$.

TCM network pharmacology is a systematic approach that combines target profile prediction of herbal compounds with 
various analytical methods to identify connections between various diseases and TCM formulas on the basis of molecular networks and to determine the active compounds and their pharmacological mechanisms. This approach is considered to be a promising way to unveil properties of TCMs and to provide valuable insights into current drug discovery and development ${ }^{[9-11]}$. Target profile prediction is one of the critical procedures in TCM network pharmacology. A powerful and recently developed method, drugCIPHER, has exhibited good performance in achieving this goal ${ }^{[12]}$. The purpose of drugCIPHER is to predict the target profile of any given compound by using linear regression models to relate drug therapeutic similarities (drugCIPHER-TS), chemical similarities (drugCIPHER-CS) and their combinations to the relevance of the targets, on the basis of a protein-protein interaction network. In previous studies, target profiles of herbal compounds predicted by drugCIPHER have been used to search for new potential uses for known bioactive compounds. One example is the identification of vitexicarpin from Vitex rotundifolia Lf (Lamiaceae) as a potential anti-angiogenesis agent ${ }^{[13]}$. Vitexicarpin has been found to specifically target AKT and SRC in the VEGF pathway ${ }^{[14]}$. Another example is the identification of tetramethylpyrazine from Ligusticum wallichii Franchat as a potent compound for alleviating oxidative organ injury induced by methotrexate ${ }^{[15]}$. In this study, we utilized a clustering analysis approach based on the target profiles predicted by drugCIPHER-CS to predict several potential antitumor compounds from an herbal compound database.

As a pilot study, the anti-tumor activities and molecular mechanisms of nuciferine, an aporphine alkaloid extracted from lotus leaves, were selected for further study and validation in our investigation, on the basis of evidence from the literature and in vivo/in vitro experiments. For example, nuciferine has been reported in studies of melanoma ${ }^{[16]}$, non-small cell lung cancer ${ }^{[17]}$ and the DU-145 cell line ${ }^{[18]}$. In addition, we predicted that nuciferine and melphalan would exhibit similar anti-tumor profiles against the tested tumor cell lines. Melphalan has been shown to be effective against advanced neuroblastoma and metastatic colorectal carcinoma ${ }^{[19,20]}$. Our experimental results also demonstrated that nuciferine has a remarkable effect of inhibiting the progression of colorectal cancers and neuroblastomas, owing to its ability to reduce their viability and invasiveness. Finally, to understand the molecular mechanisms of nuciferine, we performed network target analysis ${ }^{[14,21]}$. According to the predicted results, we addressed the question of whether inhibition of AKT, PI3K or IL1B activities or expression might be associated with the antitumor activities of nuciferine. These possibilities were validated by Western blotting.

\section{Materials and methods \\ Computational prediction methods Target prediction for herbal compounds}

The drugCIPHER-CS method uses a regression model to predict the relationship between herbal compounds and target proteins by correlating the closeness of the global pharmaco- logical network (measured by drug Chemical Similarity, CS) and the global protein-protein interaction (PPI) network ${ }^{[12]}$. Here, the drugCIPHER-CS method was used for target prediction for the given herbal compounds. drugCIPHER-CS generates these target profiles, which are composed of the concordance scores between any target proteins and the given compounds, as calculated from the correlation between the compound-drug chemical similarity vectors and the drugprotein closeness vectors within the PPI network.

\section{Clustering of anti-tumor drug and herbal compound target profiles}

Our hypothesis was that drugs with similar target profiles predicted by drugCIPHER-CS would exhibit similar bioactivities. To identify novel anti-tumor herbal compounds by using TCM resources, a standard hierarchical clustering algorithm was conducted using $R$ statistic software ( $R$ version 3.1.3). The clustering coefficient threshold was set as higher than 0.5. This threshold, as an empirical value, controls the fraction of acceptable false positives that can be generated by the clustering algorithm. We performed clustering analysis of 72 known anti-tumor drug-target profiles with a compendium of herbal compound-target profiles and thereby grouped several compounds with those known anti-tumor drugs.

\section{Identification of biological activities and molecular mechanisms of nuciferine}

To identify the biological functions of nuciferine, we used the functional enrichment tool provided by the DAVID database $\mathrm{e}^{[22]}$. According to the biological functions or pathways enriched within the nuciferine target profile, the "Pathway in cancer" and chemokine signaling pathway (hsa04062) in the KEGG pathway database ${ }^{[23]}$ appeared to indicate potential targets of nuciferine. The genes or proteins involved in these two pathways were mapped in the protein-protein interaction network of an HPRD reference data set ${ }^{[24]}$. In this network, genes or proteins with a high drugCIPHER-CS score or degree of connection were selected for validation studies.

\section{Experimental validation methods Reagents}

Nuciferine, which was obtained from Solarbio (Beijing, China), was dissolved in anhydrous methanol and kept as a stock solution at $-20^{\circ} \mathrm{C}$. Next, a class II biohazard safety cabinet was purchased from ESCO Micro Pte Ltd, and an annexin V-FITC/ PI apoptosis detection kit was purchased from Vazyme Biotech. BD Matrige ${ }^{\mathrm{TM}}$ invasion chamber 24-well plates $(8.0$ micron) were obtained from BD BioCoat. RPMI-1640, DMEM, DMEM/F12, IMDM, and FBS were purchased from HyClone. Trypsin-EDTA and penicillin/streptomycin were purchased from Macgene, and MTT was purchased from Amresco. In addition, a cell-staining solution was obtained from Nalgene.

\section{Cell culture}

All of the cell lines used in this study were purchased from the Cell Resource Center, IBMS, CAMS/PUMC. The A549 cell line 
was cultured in DMEM/F12 medium. The CT26, HT29 and NCI-H1650 cell lines were cultured in RPMI-1640 medium, and the HCT116 cell line was cultured in IMDM medium. The SY5Y cell line was cultured in DMEM/high-glucose medium. All of the cell media contained 10\% FBS (Gibco), 100 units/mL penicillin, and $100 \mu \mathrm{g} / \mathrm{mL}$ streptomycin. All of the cell lines were grown at $37^{\circ} \mathrm{C}$ with $5 \% \mathrm{CO}_{2} / 95 \%$ air in a humidified atmosphere.

\section{MTT assay}

The cells (approximately $1 \times 10^{4}$ cells/well) were seeded in 96-well flat-bottom plates ( $100 \mu \mathrm{L}$ medium/well). Following a 24-h period, the medium was replaced with medium $(100 \mu \mathrm{L} /$ well) containing different concentrations of nuciferine. After $48 \mathrm{~h}, 10 \mu \mathrm{L}$ of $5 \mathrm{mg} / \mathrm{mL}$ MTT solution in $90 \mu \mathrm{L}$ of PBS was added to each well and incubated for $4 \mathrm{~h}$. Finally, the media were discarded, and $100 \mu \mathrm{L}$ of DMSO was added to each well. The absorption of the dissolved formazan crystals was measured at $570 \mathrm{~nm}$ using a Bio-Rad microplate reader. At least three of the wells were tested for each condition, and the standard deviations were calculated.

\section{Invasion assay}

Corning BioCoat Matrigel invasion chambers were used to assess the inhibitory effects of nuciferine on tumor cell invasion ability. Approximately $5 \times 10^{4}$ cells were seeded in the upper compartment, with or without nuciferine. Medium containing $10 \% \mathrm{FBS}$ was placed in the lower chamber. The plates were then incubated at $37^{\circ} \mathrm{C}$ with $5 \% \mathrm{CO}_{2} / 95 \%$ air in a humidified atmosphere for $24 \mathrm{~h}$. The cells were fixed with $100 \%$ methanol at $4{ }^{\circ} \mathrm{C}$ and then stained with $2 \%$ crystal violet. At this point, the cells on the upper surface of the membrane were removed via scrubbing with a cotton swab, and the cells that had penetrated through the Matrigel to the lower surface of the membrane were counted at $200 \times$ magnification using a light microscope. In total, three independent experiments were conducted for this assay.

\section{Western blotting}

For the purpose of detecting the predicted targets of nuciferine, IL1B, PI3K, and other potential targets were investigated as follows: For the Western blotting experiments, SY5Y cells and CT26 cells were plated at a density of $2 \times 10^{6}$ cells/well on 6-well tissue culture plates with DMEM or RPMI-1640 growth medium supplemented with $10 \%$ FBS, respectively. After $24 \mathrm{~h}$, the wells were incubated with various concentrations of nuciferine at $37^{\circ} \mathrm{C}$. Following an incubation period of $24 \mathrm{~h}$, proteins from the cells were harvested with RIPA lysis buffer and then analyzed by Western blotting. The following antibodies were used: anti-PI3K, anti-AKT, anti-pAKT, and antiIL1-beta. In addition, beta-actin was used as the loading control.

\section{Mouse xenograft model}

CT26 cells and SY5Y cells $\left(1 \times 10^{7}\right.$ cells/body $)$ were subcutaneously implanted into the right hind paws of nude mice $(8$ to 12 weeks old, 15 to $20 \mathrm{~g}$ ) obtained from the Experimental Animal Center of the Academy of Military Medical Sciences. The tumor-bearing mice were randomly divided into three groups (5 mice per group), and the mice in each group were then injected intraperitoneally with different treatments every other day until the end of the study period. After $21 \mathrm{~d}$, the tumors were excised and weighed, and each tumor volume was measured. All animal procedures were performed with the approval of the Animal Ethics Committee of Tsinghua University.

\section{Statistical analysis}

Student's $t$-tests were used to analyze the significance of the differences between the treated and control groups. All of the data are presented as the mean values with standard deviation (SD), where $P<0.05$ was considered a significant difference when compared with controls.

\section{Results}

In silico screening of herbal compounds with anti-tumor activities In this study, a computational workflow based on TCM network pharmacology methods was applied to screen for antitumor compounds from herbs. As shown in Figure 1, the target profiles of herbal compounds and 72 FDA-approved antitumor drugs were first predicted. After determining the target profiles, we performed target profile clustering analysis of the herbal compounds and anti-tumor drugs. The herbal compounds classified within the same cluster as the 72 anti-tumor drugs were selected as the candidate active compounds. Disrupting the obesity-cancer link may represent key prevention and therapeutic targets ${ }^{[25]}$. Here, we focused our investigation on the anti-tumor activities of nuciferine, which is isolated from Nelumbo nucifera Gaertn and is an important raw material in food products intended for weight loss. Therefore, this study was primarily intended as a pilot study to evaluate the applicability of TCM network pharmacology for future discoveries.

Target profiling of small molecules by experimental methods can be used to unravel promiscuous properties with regard to their biological targets and effects ${ }^{[26]}$. Previously, we have suggested that target profiles predicted using computational approaches (eg, drugCIPHER-CS) might also help to solve this problem, as has been demonstrated elsewhere ${ }^{[14,15]}$. To computationally determine the target-specific effects derived from nuciferine treatment, we mapped the target profile of nuciferine on signaling pathways from the KEGG database and biological processes from gene ontology tools ${ }^{[27]}$ and diseases in $\mathrm{OMIM}^{[28]}$. As shown in Table 1, certain signaling pathways, biological processes or diseases enriched in the nuciferine target profile were supported by data in the literature. For example, an enriched GO term "GO:0060191 regulation of lipase activity" in the nuciferine target profile corresponded to obesity, hyperlipidemia and lipid metabolism-related processes, consistently with the lipid-lowering and weight-loss effects of nuciferine ${ }^{[29]}$. Before the study that demonstrated the nuciferine-mediated inhibition of nicotine-induced tumors ${ }^{[14]}$, 


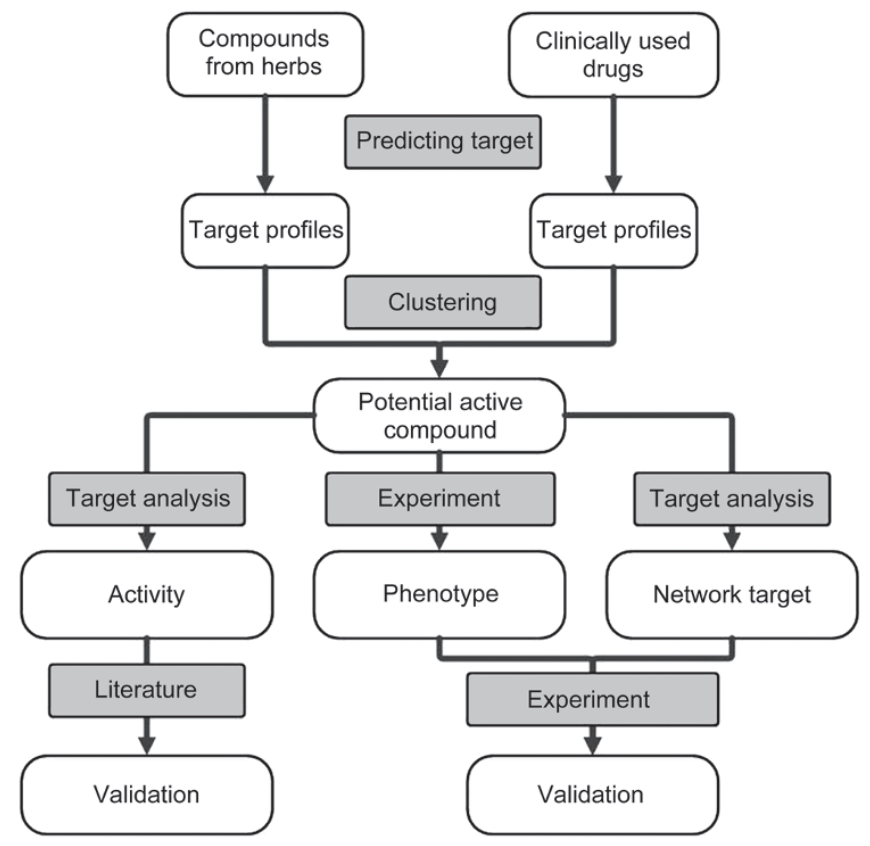

Figure 1. A network pharmacology workflow for screening active compounds from TCM herbs.

the nuciferine target profile had independently predicted "GO:0035094 response to nicotine", thus suggesting that nuciferine might interact with nicotine. Moreover, CFTR (rank 246) in 3840 previously reported druggable target genes has been shown to be activated by nuciferine ${ }^{[30]}$.

To further investigate the anti-tumor activities of nuciferine, we examined the target profile clustering results of nuciferine and 72 anti-tumor drugs (Figure 2). The therapeutic effects of characterized drugs within a given cluster might suggest hypothetical effects for the previously uncharacterized nuciferine within the same cluster (eg, anti-tumor profiles). Therefore, our results reveal that the anti-tumor effects of nuciferine treatment might be similar to those observed with melphalan. As previously reported ${ }^{[19]}$, the alkylating drug melphalan is routinely used in high-dose standard treatments for children with advanced neuroblastoma and colorectal cancer. There was a possibility that nuciferine might elicit stronger antitumor effects against neuroblastomas and colorectal cancers compared with various other cancer types. The results of the following experimental study supported this hypothesis.
Assessment of the effects of nuciferine in tumor cell proliferation and invasion assays and an in vivo tumor model

According to the anti-tumor drug screening in vitro model, the effects of nuciferine were assessed by cancer cell proliferation and invasion assays. We successfully validated our predicted results, which were consistent with the above hypothesis. Our results suggested that nuciferine, at $0.8 \mathrm{mg} / \mathrm{mL}$, elicited a more significant inhibitory effect on the proliferation of CT26 and SY5Y cells than on other cancer cells (Figure 3). We also found that cell viability decreased significantly with a higher dose $(1 \mathrm{mg} / \mathrm{mL})$ of nuciferine in SY5Y, NCI-H1650 and CT26 cells. In addition, we further investigated the effects of nuciferine on tumor cell invasion. As shown in Figure 4, the treatment of all different cancer cells with nuciferine efficiently inhibited tumor cell invasion at a specific concentration. We next used a tumor xenograft model in nude mice. CT26 and SY5Y cells were subcutaneously implanted into the right hind paws of nude mice. The tumor-bearing mice were exposed to nuciferine in the following two ways: nuciferine (1) and nuciferine (2), as shown in Figure 5. Compared with those in the control group, the tumor weights in the nuciferine (2) group were significantly reduced $(P<0.01)$. In addition, the tumor weights in the nuciferine (1) group were relatively smaller than in those in the nuciferine (2) group. Together, the results of a 6 cell-line examination panel consisting of A549, NCIH1650, CT26, HT29, HCT116 and SY5Y, as well as a tumor xenograft model, were consistent with the hypothesis that nuciferine has an anti-tumor profile similar to that of melphalan.

\section{Network analysis and experimental validation of the anti-tumor mechanisms of nuciferine}

The potent anti-tumor activity (CT26 and SY5Y) of nuciferine prompted us to investigate how it modulates cancer cell proliferation and invasion. According to the target profile of nuciferine, we sought to use network pharmacology analysis ${ }^{[8]}$ to address the following two questions arising from the list of the nuciferine target profile: what are the functional relationships among these target molecules, and what are the relationships between the target molecules and disease genes? One approach has been used to create the target network of nuciferine that is associated with colorectal cancer and neuroblastoma. This approach starts with a large network of all known interactions in the human species, which is then limited by using the genes associated with the diseases $(e g$, colorectal

Table 1. The enriched function analysis of nuciferine based on target profiles and supporting literature evidence.

\begin{tabular}{lll}
\hline Enriched functional annotation in target profile & Activities of nuciferine in the literature & References \\
\hline Regulation of lipase activity & Lowering blood lipids and inhibiting weight gain & [35] \\
Response to nicotine & Inhibiting tumor-promoting effect of nicotine & [17] \\
Chloride channel & Impacting chloride ion channel via CFTR & [36] \\
Cardiovascular diseases & Regulating cardiovascular system & [37] \\
Oxidative damage & Antioxidant effect & [38] \\
\hline
\end{tabular}


A

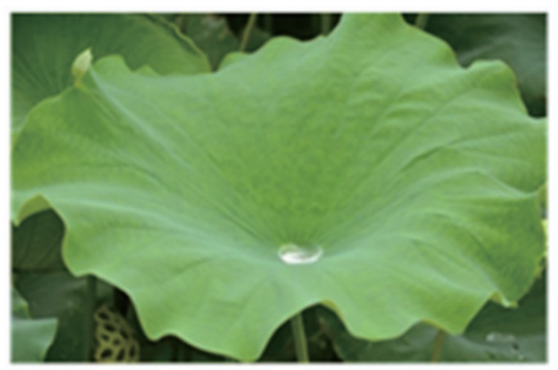

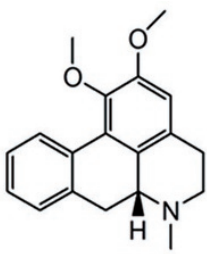

Nuciferine

Molecular formula: $\mathrm{C}_{19} \mathrm{H}_{21} \mathrm{NO}_{2}$ Molar mass: 295.376

Source: Nymphaea caerulea

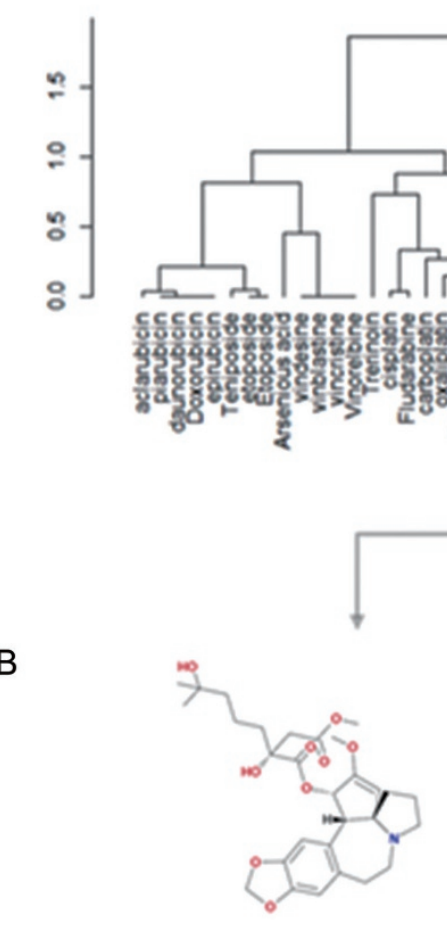

Homoharringtonine

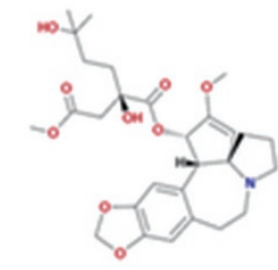

Harringtonine

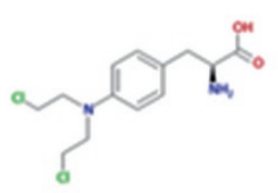

Melphalan

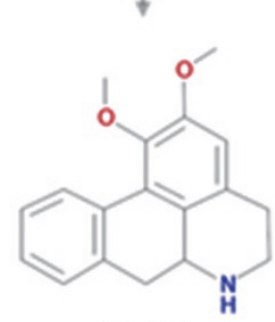

Nuciferine

Figure 2. (A) Structure information of nuciferine from lotus leaves. (B) Hierarchical clustering tree of nuciferine and 72 anti-tumor drugs.

cancer and neuroblastoma) and the nuciferine target profile as "seed nodes" to identify the largest connected subnetworks within the global network that are preferentially located near the seed nodes. Thus, we hypothesized that analysis of the target network would reveal the molecular underpinnings of nuciferine's mechanisms. As shown in Figure 6, IL1B (ranked 79 in the target profile) and PIK3CA (ranked 131) as key disease genes are highly connected nodes within the nuciferine network target. The experimental observations suggested that nuciferine reduced the expression of PI3K and IL1B and decreased the phosphorylation of AKT at a concentration of $200 \mu \mathrm{g} / \mathrm{mL}$ (Figure 7). Therefore, the nuciferine network target independently predicted the experimentally relevant observations.

\section{Discussion}

TCM network pharmacology provides a new perspective on the identification of active herbal compounds against specific diseases or pathological processes, as well as broader insights into the molecular mechanisms of the compounds. In this study, this approach provided some clues to the functional diversity of nuciferine that has previously been reported in the literature. Furthermore, our examination of in vitro cell proliferation and invasion assays might explain the sensitivity of different cancer cell lines to nuciferine, thus confirming that the anti-tumor activity of nuciferine is selective for SY5Y and CT26. The PI3K/AKT pathway is one of the most prominent pro-tumorigenesis signaling cascades that are aberrantly activated in a variety of human cancers. Recent data have demonstrated that aberrant activation of the PI3K/AKT pathway is also frequently implicated in the development of neuroblastoma and correlates with poor prognosis ${ }^{[31]}$. IL- $1 \beta$ is a pleiotropic pro-inflammatory cytokine, and its levels are increased in many cancers, including colon cancers ${ }^{[32]}$ and hepatocarcinomas $^{[33]}$. Interestingly, PI3K and IL1B are located within a hub region in the connected subnetwork that is targeted by nucif- 

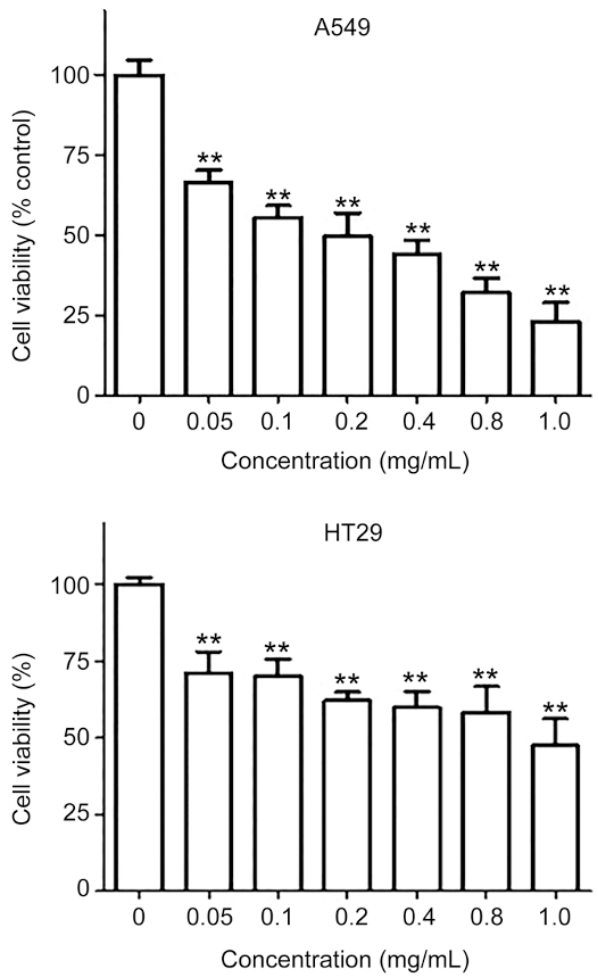

HCT116

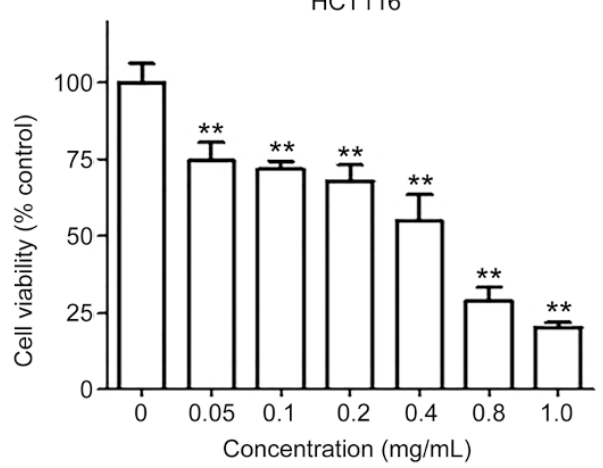

СТ26

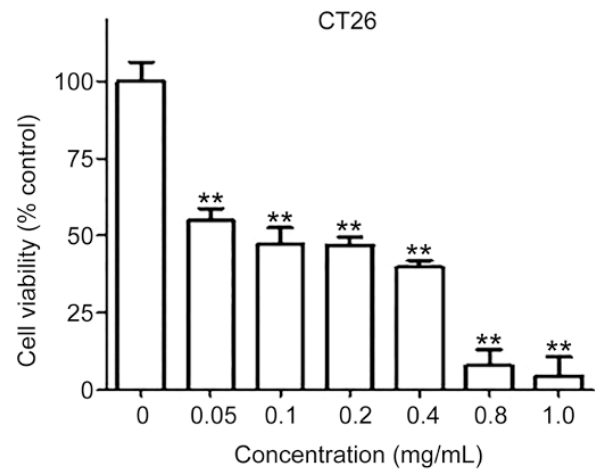

SY5Y

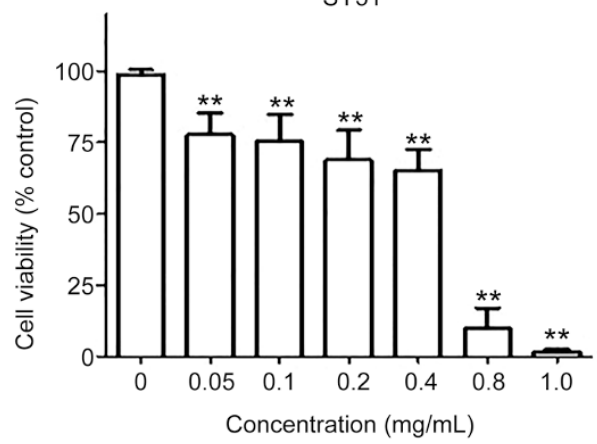

$\mathrm{NCl}-\mathrm{H} 1650$

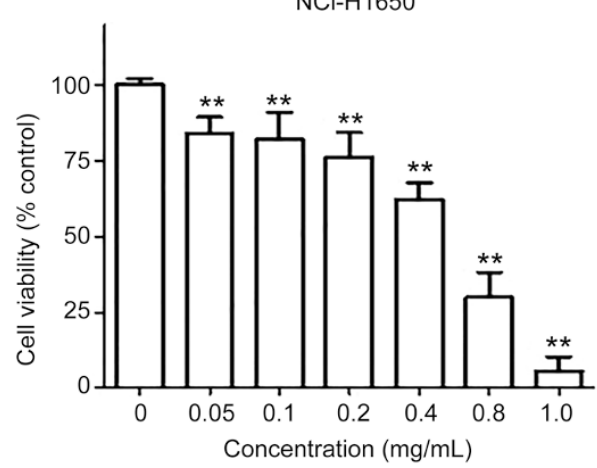

Figure 3. Cells (A549, NCl-H1650, HT29, CT26, HCT116 and SY5Y) were treated with nuciferine $(0,0.05,0.1,0.2,0.4,0.8$, and 1.0 mg/mL) for 24 h; then, viable cells were determined using an MTT assay. All data are presented as the mean \pm SD. ${ }^{* *} P<0.01$ represent a significant difference compared with the control.

erine. Our results also suggested that nuciferine can directly target the neuroblastoma- or colorectal cancer-associated proteins. Therefore, nuciferine may potentially affect the activities or expression of these gene products directly or indirectly, leading to tumor regression.

The biological significance of the results obtained through clustering analyses of "profile data" generated by computational predictions and high-throughput experiments have been demonstrated in many studies ${ }^{[34]}$. Because the predicted target profiles used in our study lacked signal (ie, activation or inhibition) and interaction strength (ie, strong or weak binding), these profiles may suggest the likelihood and importance of any target proteins affected by a given herbal compound but cannot predict whether a compound would have an active or inhibitory response against a target protein. Such information is likely to be valuable for improving the predictions. Despite this limitation, we obtained interesting evidence for the anti-tumor activity (tumor sensitivity) and mechanisms of nuciferine by using a clustering analysis of target profiles in this study. This analysis has also been used to reveal novel biological functions and mechanisms of action of tetramethylpyrazine and vitexicarpin in our previous studies. Therefore, these results suggest that an integrative method through target profile prediction and clustering analysis can be used in several ways, such as in the identification of potential active compounds in herbal compound databases and in screening for new potential uses of known herbal medicine bioactive compounds. 




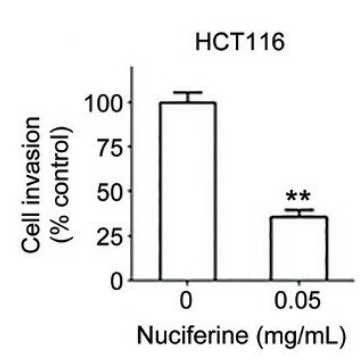
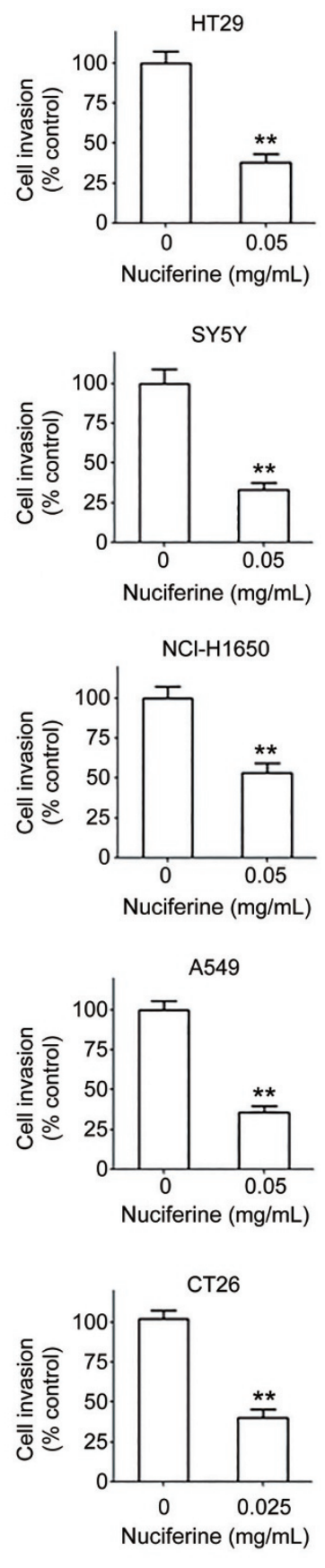

Figure 4. A Matrigel invasion chamber was used to analyze the effect of nuciferine on the invasion of A549, NCl-H1650, HT29, CT26, HCT116 and SY5Y cells. Cells that penetrated through the Matrigel to the lower surface of the filter were stained with crystal violet and imaged using a light microscope at $200 \times$ magnification. Quantification of invasion was performed by counting cells at $200 \times$. The data are presented as the mean of three independent experiments. ${ }^{* *} P<0.01$ represent a significant difference between the nuciferine treatment and the control. 

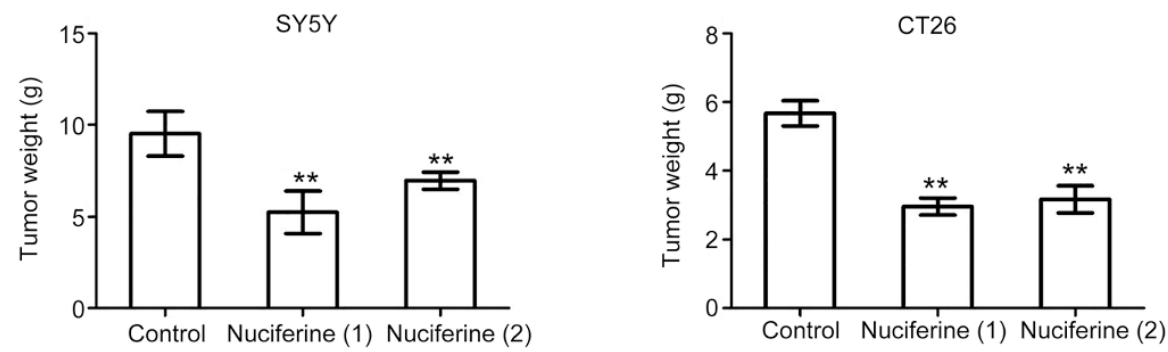

Figure 5. Mice in the nuciferine (1) group were immediately administered nuciferine (ip) after tumor implantation, and mice in the nuciferine (2) group were administered nuciferine (ip) when the tumor xenografts reached a size of $100 \mathrm{~mm}^{3}$. Mice in the nuciferine (1) group and nuciferine (2) group were administered nuciferine (ip) three times a week at a dosage of $9.5 \mathrm{mg} / \mathrm{kg}$. The tumor xenografts were removed and weighed at the end of the experiment. ${ }^{* *} P<0.01$ represents a significant difference compared with the control.<smiles>COc1cc2c3c(c1OC)-c1ccccc1C[C@H]3N(C)CC2</smiles>

Nuciferine Molecular formula: $\mathrm{C}_{19} \mathrm{H}_{21} \mathrm{NO}_{2}$ Molar mass: 295.376 Source: Nymphaea caerulea

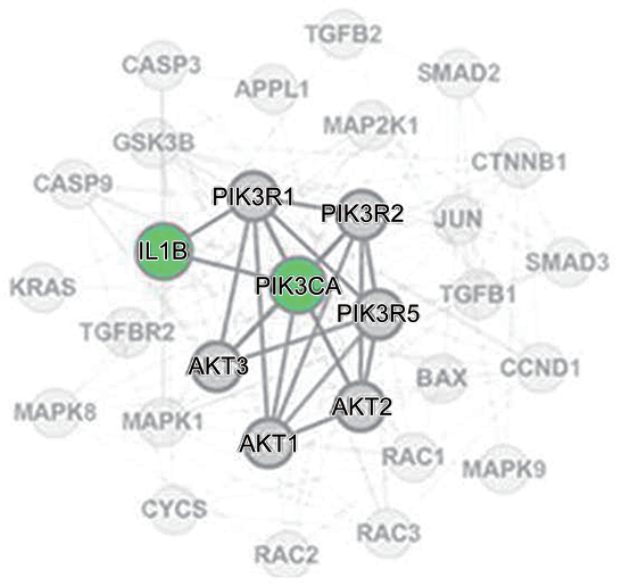

Target network of nuciferine

Figure 6. Predicted network target of nuciferine. Green nodes (IL1B, PIK3CA) are directly predicted targets, and other nodes are in the PPI network (STRING, edge value cutoff of 0.8) and CRC pathway (KEGG). The highlighted core targets were used for experimentation.

Nelumbo nucifera leaf extract has been demonstrated to possess anti-obesity and antioxidant properties ${ }^{[35,36]}$ and is commonly used for clearing heat, alleviating heatstroke and cooling and stanching blood. Nuciferine is one of the main active components of lotus leaves. Consistently with these observations, the findings obtained from our enrichment analysis based on the nuciferine target profile have implications for putative biological functions such as weight loss. Through both computational and experimental studies, we further revealed the therapeutic effects of nuciferine on cancers such as neuroblastoma and colorectal cancer, and these results might allow the traditional applications of Nelumbo nucifera to be extended. This inhibitory effect of nuciferine on neuroblastoma should be further explored to elucidate potentially uncharacterized mechanisms of neuroblastoma progression.

\section{Conclusion}

In summary, the data in this study support the feasibility of TCM network pharmacology for high-throughput activity screening. Although the anti-tumor activity of nuciferine was not strong compared with the activity of other anti-tumor drugs, our results suggest that neuroblastoma and colorectal cancer are two nuciferine-sensitive cancer types. Considering the clinical status of neuroblastoma treatment, new therapeutic agents are urgently needed, and nuciferine deserves further assessment.

\section{Acknowledgements}

This work was supported by the National Natural Science Foundation of China (№ 81225025, 81303139 and 91229201).

\section{Author contribution}

Shao LI designed the study; Quan QI, Rui LI, Yu-bing CAO, Hui-ying LI, Shu-yan WANG, and Xiao-jing FAN conducted the experiments; Quan QI, Rui LI, Ming BAI, Hui-ying LI, Bo ZHANG, and Shao LI analyzed the data; and Quan QI, Rui LI, Ming BAI, Bo ZHANG, and Shao LI wrote the paper.

\section{References}

1 Posner GH, McRiner AJ, Paik IH, Sur S, Borstnik K, Xie S, et al. Anticancer and antimalarial efficacy and safety of artemisinin-derived trioxane dimers in rodents. J Med Chem 2004; 47: 1299-301. 

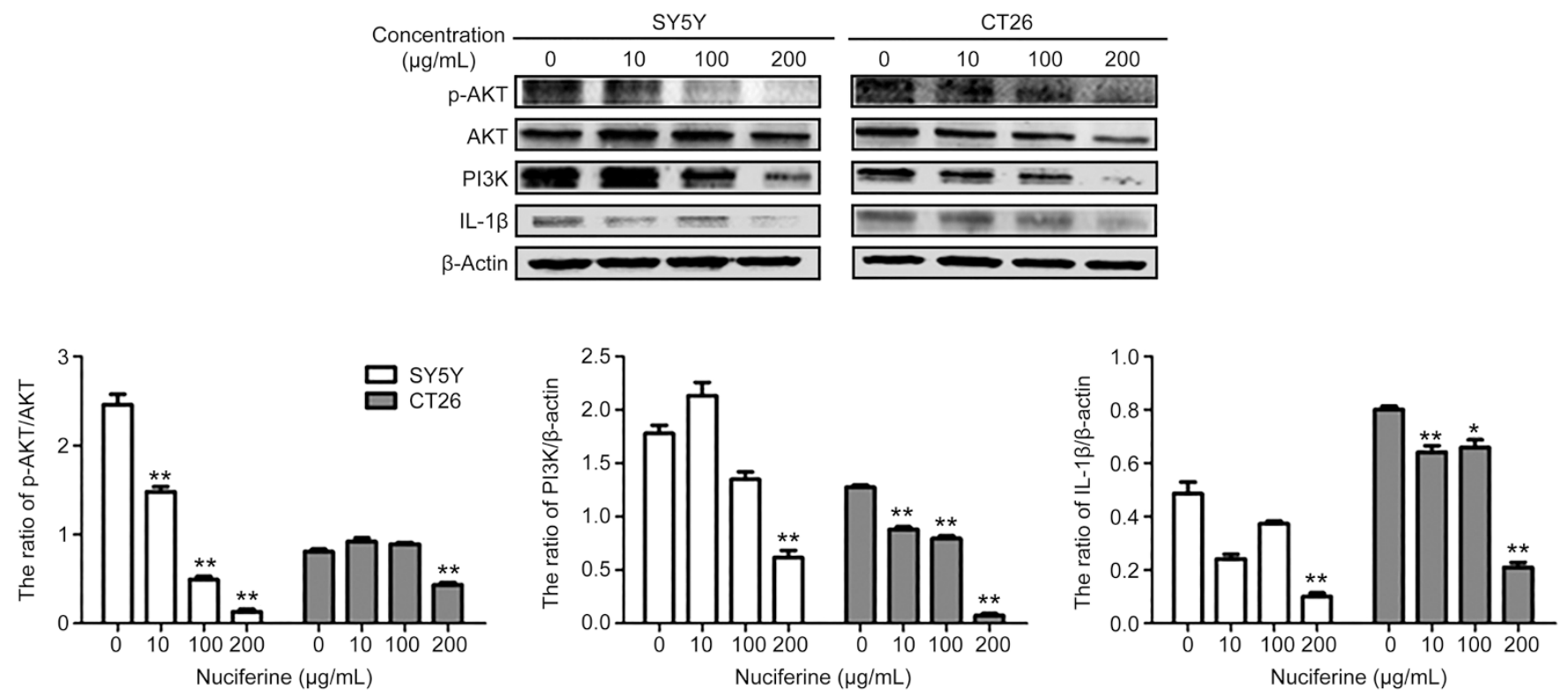

Figure 7. HCT116 cells and SY5Y cells were cultured in an atmosphere of $5 \% \mathrm{CO}_{2}$ at $37^{\circ} \mathrm{C}$. After $24 \mathrm{~h}$, the cells were treated with different concentrations of nuciferine for $24 \mathrm{~h}$. Then, proteins were extracted, and the predicted target proteins of nuciferine were detected by Western blotting. In both the HCT116 and CT26 cell lines, nuciferine inhibited the expression of p-AKT, PI3K and IL-1 $\beta$ in a dose-dependent manner. * $P<0.05, * * P<0.01$ represents a significant difference compared with the control.

2 Soignet SL, Maslak P, Wang ZG, Jhanwar S, Calleja E, Dardashti L, et al. Complete remission after treatment of acute promyelocytic leukemia with arsenic trioxide. N Engl J Med 1998; 339: 1341-8.

3 Wani MC, Taylor HL, Wall ME, Coggon P, McPhail AT. Plant antitumor agents. VI. The isolation and structure of taxol, a novel antileukemic and antitumor agent from Taxus brevifolia. J Am Chem Soc 1971; 93: 2325-7.

4 Bai F, Xu Y, Chen J, Liu Q, Gu J, Wang X, et al. Free energy landscape for the binding process of Huperzine A to acetylcholinesterase. Proc Natl Acad Sci U S A 2013; 110: 4273-8.

5 Ohlson S. Designing transient binding drugs: a new concept for drug discovery. Drug Discov Today 2008; 13: 433-9.

6 Hsiao WL, Liu L. The role of traditional Chinese herbal medicines in cancer therapy-from TCM theory to mechanistic insights. Planta Med 2010; 76: 1118-31.

7 Hopkins AL. Network pharmacology: the next paradigm in drug discovery. Nat Chem Biol 2008; 4: 682-90.

8 Li S, Zhang B. Traditional Chinese medicine network pharmacology: theory, methodology and application. Chin J Nat Med 2013; 11: 110-20.

9 Li S. Framework and practice of network-based studies for Chinese herbal formula. Zhong Xi Yi Jie He Xue Bao 2007; 5: 489-93.

10 Wu XM, Wu CF. Network pharmacology: a new approach to unveiling Traditional Chinese medicine. Chin J Nat Med 2015; 13: 1-2.

$11 \mathrm{Li} \mathrm{S}$. Mapping ancient remedies: applying a network approach to traditional Chinese medicine. Science 2015; 350: S72-4.

12 Zhao S, Li S. Network-based relating pharmacological and genomic spaces for drug target identification. PLoS One 2010; 5: e11764.

13 Li S, Zhang B, Jiang D, Wei Y, Zhang N. Herb network construction and co-module analysis for uncovering the combination rule of traditional Chinese herbal formulae. BMC Bioinformatics 2010; 11: S6.

14 Zhang B, Liu L, Zhao S, Wang X, Liu L, Li S. Vitexicarpin acts as a novel angiogenesis inhibitor and its target network. Evid Based Complement Alternat Med 2013; 2013: 278405.
15 Zhang B, Lu C, Bai M, He X, Tan Y, Bian Y, et al. Tetramethylpyrazine identified by a network pharmacology approach ameliorates methotrexate-induced oxidative organ injury. J Ethnopharmacol 2015; 175: 638-47.

16 Nakamura S, Nakashima S, Tanabe G, Oda Y, Yokota N, Fujimoto K, et al. Alkaloid constituents from flower buds and leaves of sacred lotus (Nelumbo nucifera, Nymphaeaceae) with melanogenesis inhibitory activity in B16 melanoma cells. Bioorg Med Chem 2013; 21: 779-87.

17 Liu W, Yi DD, Guo JL, Xiang ZX, Deng LF, He L. Nuciferine, extracted from Nelumbo nucifera Gaertn, inhibits tumor-promoting effect of nicotine involving $W n t / \beta$-catenin signaling in non-small cell lung cancer. J Ethnopharmacol 2015; 165: 83-93.

18 Liu CM, Kao CL, Wu HM, Li WJ, Huang CT, Li HT, et al. Antioxidant and anticancer aporphine alkaloids from the leaves of Nelumbo nucifera Gaertn. cv. Rosa-plena. Molecules 2014; 19: 17829-38.

19 Pritchard J, Cotterill SJ, Germond SM, Imeson J, de Kraker J, Jones DR. High dose melphalan in the treatment of advanced neuroblastoma: results of a randomised trial (ENSG-1) by the European Neuroblastoma Study Group. Pediatr Blood Cancer 2005; 44: 348-57.

20 Knight WA 3rd, Goodman P, Taylor SA, Macdonald JS, Coltman CA Jr, Constanzi JJ, et al. Phase II trial of intravenous melphalan for metastatic colorectal carcinoma. A Southwest Oncology Group study. Invest New Drugs 1990; 8: S87-9.

21 Zhang B, Wang X, Li S. An integrative platform of TCM network pharmacology and its application on a herbal formula, Qing-Luo-Yin. Evid Based Complement Alternat Med 2013; 2013: 456747.

22 Huang da W, Sherman BT, Lempicki RA. Bioinformatics enrichment tools: paths toward the comprehensive functional analysis of large gene lists. Nucleic Acids Res 2009; 37: 1-13.

23 Kanehisa M, Goto S, Furumichi M, Tanabe M, Hirakawa M. KEGG for representation and analysis of molecular networks involving diseases and drugs. Nucleic Acids Res 2010; 38: D355-60.

24 Keshava Prasad TS, Goel R, Kandasamy K, Keerthikumar S, Kumar S, Mathivanan S, et al. Human protein reference database -2009 
update. Nucleic Acids Res 2009; 37: D767-72.

25 Taubes G. Cancer research. Unraveling the obesity - cancer connection. Science 2012; 335: 28: 30-2.

26 Rix U, Superti-Furga G. Target profiling of small molecules by chemical proteomics. Nat Chem Biol 2009; 5: 616-24.

27 Ashburner M, Ball CA, Blake JA, Botstein D, Butler H, Cherry JM, et al. Gene ontology: tool for the unification of biology. The Gene Ontology Consortium. Nat Genet 2000; 25: 25-9.

28 Hamosh A, Scott AF, Amberger JS, Bocchini CA, McKusick VA. Online Mendelian Inheritance in Man (OMIM), a knowledgebase of human genes and genetic disorders. Nucleic Acids Res 2005; 33: D514-7.

29 Tu CC, Li XY, Yang JP, Zou L. Experimental study of lotus leaf alkaloids role in weight loss in obese rats with hyperlipidemia. J JiangXi Coll Tradit Chin Med 2001; 13: 120-1.

30 Lin S, Hou SG, Jin LL, Yu B, Yang H. Natural compound nuciferine activates chloride channel of wild type and F508 mutant cystic fibrosis transmembrane conductance regulator. Chin J Clin Pharmacol Ther 2008; 13: 138-43.

31 Fulda S. The PI3K/Akt/mTOR pathway as therapeutic target in neuroblastoma. Curr Cancer Drug Targets 2009; 9: 729-37.
32 Apte RN, Voronov E. Interleukin-1--a major pleiotropic cytokine in tumor-host interactions. Semin Cancer Biol 2002; 12: 277-90.

33 Su B, Luo T, Zhu J, Fu J, Zhao X, Chen L, et al. Interleukin-1ß/IRAK-1 inflammatory signaling contributes to persistent Gankyrin activation during hepatocarcinogenesis. Hepatology 2015; 61: 585-97.

34 Young DW, Bender A, Hoyt J, McWhinnie E, Chirn GW, Tao CY, et al. Integrating high-content screening and ligand-target prediction to identify mechanism of action. Nat Chem Biol 2008; 4: 59-68.

35 Ono Y, Hattori E, Fukaya Y, Imai S, Ohizumi Y. Anti-obesity effect of Nelumbo nucifera leaves extract in mice and rats. J Ethnopharmacol 2006; 106: 238-44.

36 Wu MJ, Wang L, Weng CY, Yen JH. Antioxidant activity of methanol extract of the lotus leaf (Nelumbo nucifera Gertn). Am J Chin Med 2003; 31: 687-98.

37 Zhang J, Xiao J, Xu K, Zhou S, Zhou Y, Wang J. The research of nuciferin's antiarrhythmic effect. Herald Med 2006; 24: 759-61.

38 Meng X, Liu XB. Inhibitory effect of tea polyphenols on Caco-2 cells and their synergistic antioxidant activity with nuciferine. Food Sci 2014; 35: 119-24. 